\title{
On the measure contraction property of metric measure spaces
}

\author{
Shin-ichi Ohta*
}

\begin{abstract}
We introduce a measure contraction property of metric measure spaces which can be regarded as a generalized notion of the lower Ricci curvature bound on Riemannian manifolds. It is actually equivalent to the lower bound of the Ricci curvature in the Riemannian case. We will generalize the Bonnet-Myers theorem, and prove that this property is preserved under the measured Gromov-Hausdorff convergence.
\end{abstract}

Mathematics Subject Classification (2000). 28C15, 53C21, 53C23.

Keywords. Measure contraction property, Ricci curvature, Bonnet-Myers theorem, GromovHausdorff convergence, Wasserstein space.

\section{Introduction}

The notions of lower and upper 'sectional' curvature bounds on not necessarily Riemannian metric spaces are introduced by Alexandrov by using the triangle comparison theorems, and they are called Alexandrov spaces and CAT $(K)$-spaces, respectively (see $[\mathrm{ABN}],[\mathrm{BGP}],[\mathrm{G}],[\mathrm{BBI}]$, and the references therein). These spaces are quite interesting objects themselves and, furthermore, they are turned out to be useful tools to study limit spaces under the Gromov-Hausdorff convergence of sequences of Riemannian manifolds with uniform lower or upper sectional curvature bounds. Now the Alexandrov spaces and CAT $(K)$-spaces are ones of the most important objects in metric geometry.

Once the importance of Alexandrov spaces and CAT $(K)$-spaces are understood, a natural question arises: What about the lower bound of the 'Ricci' curvature? One reason why this is a natural question is that the family of Riemannian manifolds with uniform lower Ricci curvature and upper diameter and dimension bounds is precompact in the Gromov-Hausdorff topology ([G]). In their serial papers [CC], Cheeger and Colding investigate the structure of limit spaces under the measured GromovHausdorff convergence of sequences of Riemannian manifolds with uniform lower

\footnotetext{
*Partially supported by the Grant-in-Aid for Scientific Research for Young Scientists (B) 16740034 from the
} Ministry of Education, Culture, Sports, Science and Technology, Japan. 
Ricci curvature bounds, and consider the convergence of the Laplacian (Fukaya's conjecture, $[\mathrm{F}])$.

One difference between the sectional and the Ricci curvatures is the role of the dimension. Sectional curvatures care only two-dimensional subspaces, so that we do not need the dimension of the entire space to define Alexandrov spaces. However, for the Ricci curvature, the dimension plays an essential role. More precisely, as a sequence of Riemannian manifolds with a uniform lower Ricci curvature bound can collapse to a lower dimensional space, we consider a combination of a lower Ricci curvature bound, say $K$, and an upper dimension bound, say $N$.

Recently, a breakthrough on this topic is given by Sturm [S2] and Lott and Villani [LV] (see also [RS]). They independently introduce mutually slightly different conditions. More precisely, they consider the Wasserstein space on a metric measure space and adopt the convexity of a (family of) functional(s) on that space as a generalized notion of the lower Ricci curvature bound. However, there remains a problem on the treatment of the dimension. Sturm's condition does not contain a term of the dimension and it can be regarded as the case of $N=\infty$. In addition to it, Lott and Villani treat the case of $N<\infty$, but only for $K=0$. So that it is still unclear how to define spaces with a finite upper bound on their dimensions and with a nonzero lower Ricci curvature bound. Furthermore, some basic questions to justify their conditions are open, for instance, whether Alexandrov spaces satisfy these or not.

In this article, we introduce another kind of generalization of the lower Ricci curvature bound, the $(K, N)$-measure contraction property (Definition 2.1, the $(K, N)$ $M C P$ for short). Here $K \in \mathbb{R}$ is the lower bound of the Ricci curvature and $N \geq 1$ is the upper bound of the dimension, so that we can consider a situation which is not covered in [S2] and [LV] $(K \neq 0$ and $N<\infty)$. This condition is defined in terms of the contraction of a measure on a set to a point, and seems simpler and more geometrically understandable. Indeed, we do not use the Wasserstein space to define the $(K, N)$-MCP, and it is not difficult to see that Alexandrov spaces satisfy the $(K, N)$-MCP (Proposition 2.8).

One of our main results is a generalization of the Bonnet-Myers theorem. Namely, we shall show that, if a metric measure space $(X, \mu)$ satisfies the $(K, N)$-MCP for some $K>0$ and $N>1$, then its diameter is less than or equal to $\pi \sqrt{(N-1) / K}$ (Theorem 4.3). Moreover, for every point $x \in X$, the set of points at a distance of $\pi \sqrt{(N-1) / K}$ from $x$ consists of at most one point (Theorem 4.5). We also prove a generalization of the Bishop-Gromov volume comparison theorem (Theorem 5.1). In addition to these, we show that, for an $n$-dimensional Riemannian manifold, the $(K, n)$-MCP is equivalent to that its Ricci curvature is bounded from below by $K$ (Theorem 3.2), and that the $(K, N)$-MCP is preserved under the measured GromovHausdorff convergence (Theorem 6.8). These results as well as the $(K, N)$-MCP of Alexandrov spaces justify us to say that the $(K, N)$-MCP is a generalized notion of 
the lower Ricci curvature bound. Techniques developed in [RS], [S2], and [LV] play crucial roles in our discussions.

The article is organized as follows. We give the definition of the $(K, N)$-MCP and consider some basic properties, such as the doubling condition, in Section 2. In Section 3, we treat the Riemannian case. Section 4 is devoted to a generalization of the Bonnet-Myers theorem. We prove a generalization of the Bishop-Gromov volume comparison theorem in Section 5. In the last section, we consider the stability of the $(K, N)$-MCP under the measured Gromov-Hausdorff convergence.

After this work was completed, I learned of a related work by Sturm [S3].

\section{Measure contraction property}

A metric space $\left(X, d_{X}\right)$ is called a length space if it satisfies $d_{X}(x, y)=\inf _{\gamma} \ell(\gamma)$ for all $x, y \in X$, where $\ell(\gamma)$ denotes the length of $\gamma$ and the infimum is taken over all rectifiable curves $\gamma$ from $x$ to $y$. If, for every $x, y \in X$, there exists a curve $\gamma$ which satisfies $d_{X}(x, y)=\ell(\gamma)$, then we say that $\left(X, d_{X}\right)$ is geodesic. Note that, if a length space is complete and locally compact, then it is geodesic. A rectifiable curve $\gamma$ in a metric space $\left(X, d_{X}\right)$ is called a geodesic if it is locally minimizing and has a constant speed. A geodesic $\gamma:[0, l] \longrightarrow X$ is said to be minimal if it satisfies $\ell(\gamma)=d_{X}(\gamma(0), \gamma(l))$. By taking a reparametrization of a curve which attains the distance, every two points in a geodesic metric space are joined by a (not necessarily unique) minimal geodesic.

Throughout this article, without otherwise indicated, let $\left(X, d_{X}\right)$ be a length space, and let $\mu$ be a Borel measure on $X$ such that $0<\mu(B(x, r))<\infty$ holds for every $x \in X$ and $r>0$, where $B(x, r)$ (or $B^{X}(x, r)$ ) denotes the open ball with center $x \in X$ and radius $r>0$. The closed ball with center $x \in X$ and radius $r>0$ is denoted by $\bar{B}(x, r)$ or $\bar{B}^{X}(x, r)$. Henceforth, we denote $d_{X}(x, y)$ by $|x-y|_{X}$ for $x, y \in X$, and write simply $X$ instead of $\left(X, d_{X}\right)$.

As in [LV], let $\Gamma$ be the set of minimal geodesics, say $\gamma:[0,1] \longrightarrow X$, in $X$ and define the evaluation map $e_{t}: \Gamma \longrightarrow X$ by $e_{t}(\gamma):=\gamma(t)$ for each $t \in[0,1]$. We regard $\Gamma$ as a subset of the set of Lipschitz maps $\operatorname{Lip}([0,1], X)$ with the uniform topology. A dynamical transference plan $\Pi$ is a Borel probability measure on $\Gamma$, and a path $\left\{\mu_{t}\right\}_{t \in[0,1]} \subset \mathscr{P}^{2}(X)$ given by $\mu_{t}=\left(e_{t}\right)_{*} \Pi$ is called a displacement interpolation associated to $\Pi$, where we define $\mathcal{P}^{2}(X)$ as the set of all Borel probability measures, say $\mu$, satisfying $\int_{X}|x-y|_{X}^{2} d \mu(y)<\infty$ for some (and hence all) $x \in X$.

For $K \in \mathbb{R}$, we define the function $s_{K}$ on $[0, \infty)($ on $[0, \pi / \sqrt{K})$ if $K>0$ ) by

$$
\boldsymbol{s}_{K}(t):= \begin{cases}(1 / \sqrt{K}) \sin (\sqrt{K} t) & \text { if } K>0, \\ t & \text { if } K=0, \\ (1 / \sqrt{-K}) \sinh (\sqrt{-K} t) & \text { if } K<0 .\end{cases}
$$


Definition 2.1. For $K, N \in \mathbb{R}$ with $N>1$, or with $K \leq 0$ and $N=1$, a metric measure space $(X, \mu)$ is said to satisfy the $(K, N)$-measure contraction property (the $(K, N)-M C P$ for short) if, for every point $x \in X$ and measurable set $A \subset X$ (provided that $A \subset B(x, \pi \sqrt{(N-1) / K})$ if $K>0$ ) with $0<\mu(A)<\infty$, there exists a displacement interpolation $\left\{\mu_{t}\right\}_{t \in[0,1]} \subset \mathscr{P}^{2}(X)$ associated to a dynamical transference plan $\Pi=\Pi_{x, A}$ satisfying the following:

(1) We have $\mu_{0}=\delta_{x}$ and $\mu_{1}=\left(\left.\mu\right|_{A}\right)^{-}$as measures, where we denote by $\left(\left.\mu\right|_{A}\right)^{-}$ the normalization of $\left.\mu\right|_{A}$, i.e., $\left(\left.\mu\right|_{A}\right)^{-}:=\left.\mu(A)^{-1} \cdot \mu\right|_{A}$;

(2) For every $t \in[0,1]$,

$$
d \mu \geq\left(e_{t}\right)_{*}\left(t\left\{\frac{s_{K}(t \ell(\gamma) / \sqrt{N-1})}{s_{K}(\ell(\gamma) / \sqrt{N-1})}\right\}^{N-1} \mu(A) d \Pi(\gamma)\right)
$$

holds as measures on $X$, where we set $0 / 0=1$ and, by convention, we read

$$
\left\{\frac{s_{K}(t \ell(\gamma) / \sqrt{N-1})}{s_{K}(\ell(\gamma) / \sqrt{N-1})}\right\}^{N-1}=1
$$

if $K \leq 0$ and $N=1$.

Remark 2.2. The case where $K>0$ and $N=1$ is an exceptional one because, by Theorem 4.3 and letting $N$ tend to 1 , then $X$ should consist of only one point. So that we do not intend to consider such a situation.

If there exists a measurable map $\Phi: A \longrightarrow \Gamma$ satisfying $e_{0} \circ \Phi \equiv x, e_{1} \circ \Phi=\mathrm{id}_{A}$, and $\Pi=\Phi_{*}\left[\left(\left.\mu\right|_{A}\right)^{-}\right]$, then the inequality (2.1) yields that

$$
d \mu \geq\left(e_{t} \circ \Phi\right)_{*}\left(t\left\{\frac{s_{K}\left(t|x-z|_{X} / \sqrt{N-1}\right)}{s_{K}\left(|x-z|_{X} / \sqrt{N-1}\right)}\right\}^{N-1} \chi_{A}(z) d \mu(z)\right)
$$

holds as measures on $X$. Here $\chi_{A}$ stands for the characteristic function on $A$. This is the case where, for each $y \in A$, there exists an exactly one geodesic $\gamma \in \operatorname{supp} \Pi$ from $x$ to $y$.

Lemma 2.3. The inequality (2.2) is equivalent to that, for all $t \in[0,1]$ and measurable sets $A^{\prime} \subset A$, we have

$$
\mu\left(e_{t}\left(\Phi\left(A^{\prime}\right)\right)\right) \geq \int_{A^{\prime}} t\left\{\frac{s_{K}\left(t|x-z|_{X} / \sqrt{N-1}\right)}{s_{K}\left(|x-z|_{X} / \sqrt{N-1}\right)}\right\}^{N-1} d \mu(z) .
$$

Proof. Put $\Psi:=e_{t} \circ \Phi$ and

$$
d \nu:=t\left\{\frac{\boldsymbol{s}_{K}\left(t|x-z|_{X} / \sqrt{N-1}\right)}{\boldsymbol{s}_{K}\left(|x-z|_{X} / \sqrt{N-1}\right)}\right\}^{N-1} \chi_{A}(z) d \mu(z)
$$


in this proof for simplicity. We first assume (2.2). For a measurable set $A^{\prime} \subset A$, we have

$$
\mu\left(\Psi\left(A^{\prime}\right)\right) \geq\left(\Psi_{*} v\right)\left(\Psi\left(A^{\prime}\right)\right)=v\left(\Psi^{-1}\left(\Psi\left(A^{\prime}\right)\right)\right) \geq v\left(A^{\prime}\right) .
$$

This implies (2.3). We next suppose (2.3). For a measurable set $W \subset X \backslash \Psi(A)$, we immediately obtain $\mu(W) \geq 0=\left(\Psi_{*} \nu\right)(W)$. If $W \subset \Psi(A)$, then (2.3) yields that

$$
\mu(W)=\mu\left(\Psi\left(\Psi^{-1}(W)\right)\right) \geq v\left(\Psi^{-1}(W)\right)=\left(\Psi_{*} \nu\right)(W) .
$$

This completes the proof.

The inequality (2.3) can be regarded as a generalization of the Bishop inequality under a lower Ricci curvature bound $\mathrm{Ric}_{g} \geq K$ (see Theorem 3.1 below), and is a reason why we say that (2.1) is a kind of measure contraction property. We refer [S1], [KS1], [R1], and [R2] (see also [O]) for other kinds of measure contraction property of metric measure spaces. Especially, an essentially similar condition to our MCP is proposed in [CC, I, Appendix 2] (see also [G]).

Lemma 2.4. (i) The $(K, N)$-MCP of $(X, \mu)$ implies the $\left(K^{\prime}, N^{\prime}\right)$-MCPfor all $K^{\prime} \leq K$ and $N^{\prime} \geq N$.

(ii) If $\left(X, d_{X}, \mu\right)$ satisfies the $(K, N)-M C P$ and if $a, b>0$, then the scaled metric measure space $\left(X, a \cdot d_{X}, b \cdot \mu\right)$ satisfies $\left(K / a^{2}, N\right)$-MCP.

Proof. (i) By calculation, we see that

$$
\left\{\frac{s_{K}(t d / \sqrt{N-1})}{s_{K}(d / \sqrt{N-1})}\right\}^{N-1}
$$

is monotone non-decreasing in $K$ for any fixed $N>1$, and is monotone non-increasing in $N$ for any fixed $K \in \mathbb{R}$. This (together with Theorem 4.3 and Lemma 4.4 (i) if $K>0$ ) completes the proof.

(ii) It is clear by the definition of the $(K, N)$-MCP.

The following lemma is straightforward from the definition of the $(K, N)$-MCP, and will be sharpened in Section 5 .

Lemma 2.5. Suppose that $(X, \mu)$ satisfies the $(K, N)$-MCP. Then, for every $x \in X$ and $0<r \leq R(\leq \pi \sqrt{(N-1) / K}$ if $K>0)$, we have

$$
\frac{\mu(B(x, R))}{\mu(B(x, r))} \leq \frac{R}{r} \sup _{0 \leq \lambda \leq 1}\left\{\frac{s_{K}(\lambda R / \sqrt{N-1})}{s_{K}(\lambda r / \sqrt{N-1})}\right\}^{N-1} .
$$

In particular, the set $S(x, r):=\left\{y \in X|| x-\left.y\right|_{X}=r\right\}$ has a null measure for any $x \in X$ and $r>0$ (provided that $r<\pi \sqrt{(N-1) / K}$ if $K>0$ ). 
Proof. The $(K, N)$-MCP with $x=x, A=B(x, R)$, and $t=r / R$ yields that

$$
\begin{aligned}
\mu & B(x, r)) \\
& \geq \mu(B(x, R)) \frac{r}{R} \inf _{0 \leq \lambda \leq 1}\left\{\frac{s_{K}(\lambda r / \sqrt{N-1})}{s_{K}(\lambda R / \sqrt{N-1})}\right\}^{N-1}\left(\left(e_{r / R}\right)_{*} \Pi_{x, B(x, R)}\right)(B(x, r)) \\
& =\mu(B(x, R)) \frac{r}{R} \inf _{0 \leq \lambda \leq 1}\left\{\frac{s_{K}(\lambda r / \sqrt{N-1})}{s_{K}(\lambda R / \sqrt{N-1})}\right\}^{N-1} \Pi\left(\left(e_{r / R}\right)^{-1}[B(x, r)]\right) \\
& =\mu(B(x, R)) \frac{r}{R} \inf _{0 \leq \lambda \leq 1}\left\{\frac{s_{K}(\lambda r / \sqrt{N-1})}{s_{K}(\lambda R / \sqrt{N-1})}\right\}^{N-1} \Pi(\operatorname{supp} \Pi) \\
& =\frac{r}{R} \inf _{0 \leq \lambda \leq 1}\left\{\frac{s_{K}(\lambda r / \sqrt{N-1})}{s_{K}(\lambda R / \sqrt{N-1})}\right\}^{N-1} \mu(B(x, R)) .
\end{aligned}
$$

Here the third equality follows from $\left(e_{0}\right)_{*} \Pi=\delta_{x}$ and $\left(e_{1}\right)_{*} \Pi=\left(\left.\mu\right|_{B(x, R)}\right)^{-}$. Indeed, it implies $e_{r / R}(\gamma) \in B(x, r)$ for $\Pi$-a.e. $\gamma$. This completes the proof.

In particular, the $(K, N)$-MCP implies the (local) doubling condition. Namely, for any $R>0(R \leq \pi \sqrt{(N-1) / K}$ if $K>0), r \in(0, R]$, and $x \in X$, we have

$$
\frac{\mu(B(x, r))}{\mu(B(x, r / 2))} \leq C_{K, N, R}
$$

where $C_{K, N, R}<\infty$ is a constant depending only on $K, N$, and $R$. The doubling condition implies that every bounded closed ball in $X$ is totally bounded. Therefore, if $X$ is complete, then it is proper (i.e., all bounded closed sets are compact) and hence geodesic.

Corollary 2.6. If $(X, \mu)$ satisfies the $(K, N)-M C P$ and if it contains more than two points, then the measure $\mu$ is non-atomic.

Corollary 2.7. If $(X, \mu)$ satisfies the $(K, N)-M C P$, then the Hausdorff dimension of $X$ is less than or equal to $N$.

Proof. Lemma 2.5 yields that the function $f(x):=\limsup _{r \rightarrow 0} r^{N} \mu(B(x, r))^{-1}$ on $X$ is locally bounded. By [AT, Theorem 2.4.3], this implies that the $N$-dimensional Hausdorff measure $\mathscr{H}^{N}$ on $X$ is also locally bounded. Therefore the Hausdorff dimension of $X$ is not greater than $N$.

We end this section with a proposition which asserts that Alexandrov spaces satisfy the MCP. As the Alexandrov space is considered as a metric space with a lower 'sectional' curvature bound, this proposition supports us for saying that the $(K, N)$-MCP is a generalized notion of a lower 'Ricci' curvature bound. See [BBI], [BGP], and [KS1] for the definition of and terminologies on Alexandrov spaces. 
Proposition 2.8. Let $X$ be an n-dimensional, complete Alexandrov space with curvature $\geq K$, and $\mathscr{H}^{n}$ be the $n$-dimensional Hausdorff measure on $X$. Then $\left(X, \mathscr{H}^{n}\right)$ satisfies the $((n-1) K, n)-M C P$.

Proof. This easily follows from [KS1, Lemma 6.1], we give an outline of the proof for completeness. For a point $x \in X$ and a measurable set $A \subset X$, we define a map $\Phi^{X}=\Phi_{x, A}^{X}: A \longrightarrow \Gamma$ by $\Phi^{X}(y):=\gamma$, where $\gamma:[0,1] \longrightarrow X$ is an arbitrarily chosen minimal geodesic from $x$ to $y$. Then we see that $\Phi^{X}$ is measurable as in the proof of [KS1, Proposition 6.1], and we put $\Pi:=\left(\Phi^{X}\right) *\left[\left(\left.\mu\right|_{A}\right)^{-}\right]$. The condition (1) in Definition 2.1 is clearly satisfied and the condition (2) follows from the curvature condition just as in [KS1, Lemma 6.1].

\section{Riemannian case}

In this section, we consider the Riemannian case. See, for example, $[\mathrm{Cl}]$ for fundamentals on Riemannian geometry. Let $(M, g)$ be an $n$-dimensional, complete Riemannian manifold without boundary and denote by $d_{g}$ (or $|\cdot-\cdot|_{g}$ ) and $v_{g}$ the Riemannian distance and the Riemannian measure, respectively, on $M$ induced from $g$. In addition, $\mathrm{Ric}_{g}$ stands for the Ricci tensor with respect to $g$ and the inequality $\operatorname{Ric}_{g} \geq K$ means that $\operatorname{Ric}_{g}(\xi, \xi) \geq K$ holds for every $p \in M$ and $\xi \in S_{p} M$, where $S_{p} M \subset T_{p} M$ is the unit tangent sphere at $p \in M$. For a point $p \in M$ and a unit tangent vector $\xi \in S_{p} M$, we set

$$
c(\xi):=\sup \left\{r>0|| p-\left.\gamma_{\xi}(r)\right|_{g}=r\right\},
$$

where we define $\gamma_{\xi}(r):=\exp _{p} r \xi$. Define, for $p \in M$,

$$
\begin{aligned}
& C(p):=\left\{\gamma_{\xi}(c(\xi)) \mid \xi \in S_{p} M\right\}, \\
& \mathrm{D}(p):=\left\{r \xi \mid \xi \in S_{p} M, 0 \leq r<c(\xi)\right\} \subset T_{p} M, \\
& D(p):=\exp _{p} \mathrm{D}(p) .
\end{aligned}
$$

The set $C(p)$ is called the cut locus of $p$. Recall that $\exp _{p}: \mathrm{D}(p) \longrightarrow D(p)$ gives a diffeomorphism and that we can represent $d v_{g}(q)=\left(\exp _{p}\right)_{*}\left[\mathrm{~A}_{p}(r ; \xi) d r d \xi\right]$ on $D(p)$, where $q=\gamma_{\xi}(r)$ and $\mathrm{A}_{p}(r ; \xi)$ denotes the density of the Riemannian measure on $S(p, r)$ induced from $g$. Recall that we set $S(p, r):=\left\{q \in M|| p-\left.q\right|_{g}=r\right\}$. The classical Bishop comparison theorem asserts the following ([BC], cf. [Cl, Theorem 3.8]).

Theorem 3.1. If $(M, g)$ satisfies $\operatorname{Ric}_{g} \geq K$, then we have

$$
\frac{1}{\mathrm{~A}_{p}(r ; \xi)} \frac{d \mathrm{~A}_{p}(r ; \xi)}{d r} \leq(n-1) \frac{\boldsymbol{s}_{K}^{\prime}(r / \sqrt{n-1})}{\boldsymbol{s}_{K}(r / \sqrt{n-1})}
$$


for all $\xi \in S_{p} M$ and $r \in(0, c(\xi))$. In particular, the function

$$
\frac{\mathrm{A}_{p}(r ; \xi)}{\boldsymbol{s}_{K}(r / \sqrt{n-1})^{n-1}}
$$

is monotone non-increasing in $r \in(0, c(\xi))$.

Given a point $p \in M$ and a measurable set $A \subset M$, as in the proof of Proposition 2.8, we define a map $\Phi^{M}=\Phi_{p, A}^{M}: A \longrightarrow \Gamma$ by $\Phi_{p, A}^{M}(q):=\gamma$, where $\gamma:[0,1] \longrightarrow M$ is an arbitrarily chosen minimal geodesic from $p$ to $q$. As $C(p)$ has a null measure, the map $\Phi_{p, A}^{M}$ is measurable and is uniquely determined up to a modification on a null measure set.

Theorem 3.2. Let $(M, g)$ be an $n$-dimensional, complete Riemannian manifold without boundary with $n \geq 2$. Then a metric measure space $\left(M, d_{g}, v_{g}\right)$ satisfies the $(K, n)-M C P$ if and only if $\operatorname{Ric}_{g} \geq K$ holds.

Proof. We first assume $\operatorname{Ric}_{g} \geq K$ and fix a point $p \in M$ and a measurable set $A \subset M$. We shall show that the map $\Phi^{M}=\Phi_{p, A}^{M}$ defined as above satisfies (2.3) with $N=n$ which implies the $(K, n)$-MCP. It follows from Theorem 3.1 that, for any $t \in[0,1]$ and measurable subset $A^{\prime} \subset A$,

$$
\begin{aligned}
v_{g}\left(e_{t}\left(\Phi_{p, A}^{M}\left(A^{\prime}\right)\right)\right) & =\int_{\exp _{p}^{-1}\left(A^{\prime}\right) \cap \mathrm{D}(p)} t \mathrm{~A}_{p}(t r ; \xi) d r d \xi \\
& \geq \int_{\exp _{p}^{-1}\left(A^{\prime}\right) \cap \mathrm{D}(p)} t\left\{\frac{\boldsymbol{s}_{K}(t r / \sqrt{n-1})}{\boldsymbol{s}_{K}(r / \sqrt{n-1})}\right\}^{n-1} \mathrm{~A}_{p}(r ; \xi) d r d \xi \\
& =\int_{A^{\prime}} t\left\{\frac{\boldsymbol{s}_{K}\left(t|p-q|_{g} / \sqrt{n-1}\right)}{\boldsymbol{s}_{K}\left(|p-q|_{g} / \sqrt{n-1}\right)}\right\}^{n-1} d v_{g}(q) .
\end{aligned}
$$

Therefore $\Phi^{M}$ satisfies the inequality (2.3).

Next we consider the converse, so that we suppose that $\left(M, d_{g}, v_{g}\right)$ satisfies the $(K, n)$-MCP. Fix $p \in M, \xi \in S_{p} M$, and an orthonormal basis $\left\{e_{1}, \ldots, e_{n}\right\}$ in $T_{p} M$ with $e_{1}=\xi$. We denote by $k_{i}$ the sectional curvature of the plane spanned by $e_{1}$ and $e_{i}$ for each $i=2, \ldots, n$. For a small $r>0$, it follows from

$$
\frac{\boldsymbol{s}_{K}(r)}{\boldsymbol{s}_{K}(2 r)}=\frac{1}{2}\left(1+\frac{K}{2} r^{2}+O\left(r^{4}\right)\right)
$$


that

$$
\begin{aligned}
\frac{\mathrm{A}_{p}(r ; \xi)}{\mathrm{A}_{p}(2 r ; \xi)} & =\frac{1}{2^{n-1}} \prod_{i=2}^{n}\left(1+\frac{k_{i}+O(r)}{2} r^{2}+O\left(r^{4}\right)\right) \\
& =\frac{1}{2^{n-1}} \prod_{i=2}^{n}\left(1+\frac{k_{i}}{2} r^{2}\right)+O\left(r^{3}\right) \\
& =\frac{1}{2^{n-1}}\left\{1+\sum_{i=2}^{n} k_{i} r^{2}\right\}+O\left(r^{3}\right) \\
& =\frac{1}{2^{n-1}}\left\{1+\operatorname{Ric}_{g}(\xi, \xi) r^{2}\right\}+O\left(r^{3}\right) .
\end{aligned}
$$

On the other hand, it is not difficult to observe that the $(K, n)$-MCP implies

$$
\frac{\mathrm{A}_{p}(r ; \xi)}{\mathrm{A}_{p}(2 r ; \xi)} \geq\left\{\frac{\boldsymbol{s}_{K}(r / \sqrt{n-1})}{\boldsymbol{s}_{K}(2 r / \sqrt{n-1})}\right\}^{n-1},
$$

and hence we have

$$
\begin{aligned}
\operatorname{Ric}_{g}(\xi, \xi) r^{2} & \geq\left\{\frac{2 s_{K}(r / \sqrt{n-1})}{s_{K}(2 r / \sqrt{n-1})}\right\}^{n-1}-1+O\left(r^{3}\right) \\
& =\left\{1+\frac{K}{n-1} r^{2}\right\}^{n-1}-1+O\left(r^{3}\right) \\
& =K r^{2}+O\left(r^{3}\right) .
\end{aligned}
$$

Dividing both sides by $r^{2}$ and letting $r$ tend to zero, we consequently obtain

$$
\operatorname{Ric}_{g}(\xi, \xi) \geq K \text {. }
$$

This completes the proof.

The following are easily derived from Lemma 2.4(i) and Corollary 2.7 together with the theorem above.

Corollary 3.3. Let $(M, g)$ be an n-dimensional, complete Riemannian manifold without boundary.

(i) If $(M, g)$ satisfies $\operatorname{Ric}_{g} \geq K$ and $n \leq N$, then $\left(M, d_{g}, v_{g}\right)$ satisfies the $(K, N)$ $M C P$.

(ii) If a metric measure space $\left(M, d_{g}, v_{g}\right)$ satisfies the $(K, N)-M C P$, then we have $n \leq N$. 


\section{Bonnet-Myers theorem}

In this section, we shall show a generalization of the Bonnet-Myers theorem ([M]), that is, the $(K, N)$-MCP with $K>0$ and $N>1$ implies that the diameter is less than or equal to $\pi \sqrt{(N-1) / K}$. By rescaling the distance, we may assume $K=N-1$ (Lemma 2.4(ii)). For $x \in X$ and $s, t \geq 0$ with $s<t$, we define $A(x ; s, t):=$ $B(x, t) \backslash B(x, s)$, where we set $B(x, 0):=\emptyset$. The symbol $\theta_{\alpha, \beta}(\delta)$ denotes a function depending only on $\alpha$ and $\beta$ with $\lim _{\delta \rightarrow 0} \theta_{\alpha, \beta}(\delta)=0$. Before beginning the proof of the Bonnet-Myers theorem, we prove a useful lemma which holds for general $K$ and $N$.

Lemma 4.1. Let $(X, \mu)$ satisfy the $(K, N)-M C P$ and, for $0 \leq r<r^{\prime} \leq \infty(0 \leq$ $r<r^{\prime} \leq \pi \sqrt{(N-1) / K}$ if $\left.K>0\right)$, let $\tau:\left(r, r^{\prime}\right) \longrightarrow(0,1]$ be a $C^{1}$-function satisfying $\tau^{\prime}(l) l+\tau(l)>0$ for all $l \in\left(r, r^{\prime}\right)$. Then we have, for any point $x \in X$, any measurable set $A \subset A\left(x ; r, r^{\prime}\right)$ with $0<\mu(A)<\infty$, and for $\Pi=\Pi_{x, A}$ as in Definition 2.1,

$$
\begin{aligned}
d \mu \geq\left(e_{\tau}\right)_{*}\left[\left\{\tau^{\prime}(\ell(\gamma)) \ell(\gamma)+\tau(\ell(\gamma))\right\}\right. & \\
& \left.\times\left\{\frac{s_{K}(\tau(\ell(\gamma)) \ell(\gamma) / \sqrt{N-1})}{s_{K}(\ell(\gamma) / \sqrt{N-1})}\right\}^{N-1} \mu(A) d \Pi(\gamma)\right]
\end{aligned}
$$

as measures. Here $e_{\tau}: \Gamma \longrightarrow X$ denotes a map defined by $e_{\tau}(\gamma):=e_{\tau(\ell(\gamma))}(\gamma)$.

Proof. Choose an arbitrary measurable set $W \subset X$. It suffices to show

$$
\begin{aligned}
\mu(W) \geq \int_{e_{\tau}^{-1}(W)}\left[\left\{\tau^{\prime}(\ell(\gamma)) \ell(\gamma)+\tau(\ell(\gamma))\right\}\right. & \\
& \left.\times\left\{\frac{s_{K}(\tau(\ell(\gamma)) \ell(\gamma) / \sqrt{N-1})}{s_{K}(\ell(\gamma) / \sqrt{N-1})}\right\}^{N-1} \mu(A)\right] d \Pi(\gamma) .
\end{aligned}
$$

In the case of $K>0$, without loss of generality, we may assume

$$
W \subset B(x, \pi \sqrt{(N-1) / K}-\varepsilon)
$$

for some $\varepsilon>0$. Take a large $M \in \mathbb{N}$, set $\delta:=\left(r^{\prime}-r\right) / M$ and $r_{m}:=r+m \delta$ for $0 \leq m \leq M$, and put

$$
\Gamma_{m}:=e_{\tau}^{-1}(W) \cap\left\{\gamma \in \Gamma \mid e_{0}(\gamma)=x, e_{1}(\gamma) \in A\left(x ; r_{m-1}, r_{m}\right)\right\}
$$

and $W_{m}:=e_{\tau}\left(\Gamma_{m}\right)$ for $1 \leq m \leq M$. The hypothesis on $\tau$ says that

$$
[\tau(l) l]^{\prime}=\tau^{\prime}(l) l+\tau(l)>0,
$$


and hence $W_{m} \cap W_{m^{\prime}}=\emptyset$ holds if $m \neq m^{\prime}$.

For each $1 \leq m \leq M$, we denote by $k_{m} \in \mathbb{N} \cup\{0\}$ the number satisfying

$$
\tau\left(r_{m}\right)\left(\frac{r_{m-1}}{r_{m}}\right)^{k_{m}+1} r_{m} \leq \tau\left(r_{m-1}\right) r_{m-1}<\tau\left(r_{m}\right)\left(\frac{r_{m-1}}{r_{m}}\right)^{k_{m}} r_{m} .
$$

Moreover, for each $1 \leq m \leq M$, we can choose $a_{m}, b_{m} \in\left[r_{m-1}, r_{m}\right]$ with $a_{m}<b_{m}$ and $k_{m}^{\prime} \geq k_{m}$ such that

$$
\tau\left(r_{m-1}\right) r_{m-1} \leq \tau\left(r_{m}\right)\left(\frac{a_{m}}{b_{m}}\right)^{k_{m}^{\prime}+1} r_{m} \leq \tau\left(r_{m-1}\right) r_{m-1}+\tau\left(r_{m}\right) \delta^{2}
$$

as well as

$$
\begin{aligned}
\int_{\Gamma_{m}^{\prime}} & \left\{\frac{s_{K}\left(\tau\left(r_{m}\right) \ell(\gamma) / \sqrt{N-1}\right)}{s_{K}(\ell(\gamma) / \sqrt{N-1})}\right\}^{N-1} d \Pi(\gamma) \\
& \geq \frac{b_{m}-a_{m}}{r_{m}-r_{m-1}} \int_{\Gamma_{m}}\left\{\frac{s_{K}\left(\tau\left(r_{m}\right) \ell(\gamma) / \sqrt{N-1}\right)}{s_{K}(\ell(\gamma) / \sqrt{N-1})}\right\}^{N-1} d \Pi(\gamma),
\end{aligned}
$$

where we put

$$
\Gamma_{m}^{\prime}:=e_{\tau}^{-1}(W) \cap\left\{\gamma \in \Gamma \mid e_{0}(\gamma)=x, e_{1}(\gamma) \in A\left(x ; a_{m}, b_{m}\right)\right\} \subset \Gamma_{m} .
$$

Note that, for all $l \in\left(a_{m}, b_{m}\right)$ and $0 \leq k \leq k_{m}^{\prime}$,

$$
\begin{aligned}
\frac{\tau\left(r_{m}\right) r_{m}}{b_{m}}\left(\frac{a_{m}}{b_{m}}\right)^{k} l & \in\left(\tau\left(r_{m}\right) r_{m}\left(\frac{a_{m}}{b_{m}}\right)^{k+1}, \tau\left(r_{m}\right) r_{m}\left(\frac{a_{m}}{b_{m}}\right)^{k}\right) \\
& \subset\left(\tau\left(r_{m-1}\right) r_{m-1}, \tau\left(r_{m}\right) r_{m}\right),
\end{aligned}
$$

and hence

$$
W_{m} \supset \bigsqcup_{k=0}^{k_{m}^{\prime}} e_{\left(\tau\left(r_{m}\right) r_{m} / b_{m}\right)\left(a_{m} / b_{m}\right)^{k}}\left(\Gamma_{m}^{\prime}\right) \quad \text { (disjoint union). }
$$


Therefore we have, by the $(K, N)$-MCP,

$$
\begin{aligned}
\mu(W) \geq & \sum_{m=1}^{M} \mu\left(W_{m}\right) \geq \sum_{m=1}^{M}\left[\sum_{k=0}^{k_{m}^{\prime}} \mu\left(e_{\left(\tau\left(r_{m}\right) r_{m} / b_{m}\right)\left(a_{m} / b_{m}\right)^{k}}\left(\Gamma_{m}^{\prime}\right)\right)\right] \\
\geq & \sum_{m=1}^{M}\left[\sum_{k=0}^{k_{m}^{\prime}} \frac{\tau\left(r_{m}\right) r_{m}}{b_{m}}\left(\frac{a_{m}}{b_{m}}\right)^{k}\right. \\
& \left.\times \int_{\Gamma_{m}^{\prime}}\left\{\frac{s_{K}\left(\left\{\tau\left(r_{m}\right)+\theta(\delta)\right\} \ell(\gamma) / \sqrt{N-1}\right)}{s_{K}(\ell(\gamma) / \sqrt{N-1})}\right\}^{N-1} \mu(A) d \Pi(\gamma)\right] \\
= & \sum_{m=1}^{M}\left[\frac{\tau\left(r_{m}\right) r_{m}}{b_{m}} \frac{1-\left(a_{m} / b_{m}\right)^{k_{m}^{\prime}+1}}{1-\left(a_{m} / b_{m}\right)}\right. \\
& \left.\times \int_{\Gamma_{m}^{\prime}}\left\{\left\{\frac{s_{K}\left(\tau\left(r_{m}\right) \ell(\gamma) / \sqrt{N-1}\right)}{s_{K}(\ell(\gamma) / \sqrt{N-1})}\right\}^{N-1}+\theta(\delta)\right\} \mu(A) d \Pi(\gamma)\right] .
\end{aligned}
$$

Furthermore, it follows from (4.2) and (4.3) that

$$
\begin{aligned}
\sum_{m=1}^{M} & {\left[\frac{\tau\left(r_{m}\right) r_{m}}{b_{m}} \frac{1-\left(a_{m} / b_{m}\right)^{k_{m}^{\prime}+1}}{1-\left(a_{m} / b_{m}\right)}\right.} \\
& \left.\times \int_{\Gamma_{m}^{\prime}}\left\{\left\{\frac{s_{K}\left(\tau\left(r_{m}\right) \ell(\gamma) / \sqrt{N-1}\right)}{\boldsymbol{s}_{K}(\ell(\gamma) / \sqrt{N-1})}\right\}^{N-1}+\theta(\delta)\right\} \mu(A) d \Pi(\gamma)\right] \\
\geq & \sum_{m=1}^{M}\left[\frac{\tau\left(r_{m}\right) r_{m}}{b_{m}} \frac{1-\left(a_{m} / b_{m}\right)^{k_{m}^{\prime}+1}}{1-\left(a_{m} / b_{m}\right)} \frac{b_{m}-a_{m}}{r_{m}-r_{m-1}}\right. \\
& \left.\times \int_{\Gamma_{m}}\left\{\left\{\frac{s_{K}\left(\tau\left(r_{m}\right) \ell(\gamma) / \sqrt{N-1}\right)}{\boldsymbol{s}_{K}(\ell(\gamma) / \sqrt{N-1})}\right\}^{N-1}+\theta(\delta)\right\} \mu(A) d \Pi(\gamma)\right] \\
\geq & \sum_{m=1}^{M}\left[\left\{\frac{\tau\left(r_{m}\right) r_{m}-\tau\left(r_{m-1}\right) r_{m-1}}{r_{m}-r_{m-1}}-\tau\left(r_{m}\right) \delta\right\}\right. \\
& \left.\times \int_{\Gamma_{m}}\left\{\left\{\frac{s_{K}\left(\tau\left(r_{m}\right) \ell(\gamma) / \sqrt{N-1}\right)}{s_{K}(\ell(\gamma) / \sqrt{N-1})}\right\}^{N-1}+\theta(\delta)\right\} \mu(A) d \Pi(\gamma)\right] \\
\rightarrow & \int_{\Gamma}\left\{\tau^{\prime}(\ell(\gamma)) \ell(\gamma)+\tau(\ell(\gamma))\right\}\left\{\frac{s_{K}(\tau(\ell(\gamma)) \ell(\gamma) / \sqrt{N-1})}{s_{K}(\ell(\gamma) / \sqrt{N-1})}\right\}^{N-1} \mu(A) d \Pi(\gamma)
\end{aligned}
$$

as $M$ diverges to the infinity. We remark that, to see

$$
\lim _{M \rightarrow \infty} \tau\left(r_{m}\right) \delta \int_{\Gamma_{m}}\left\{\frac{s_{K}\left(\tau\left(r_{m}\right) \ell(\gamma) / \sqrt{N-1}\right)}{s_{K}(\ell(\gamma) / \sqrt{N-1})}\right\}^{N-1} \mu(A) d \Pi(\gamma)=0
$$


in the last implication, we used the fact that

$$
\begin{gathered}
\int_{\Gamma} \tau(\ell(\gamma))\left\{\frac{s_{K}(\tau(\ell(\gamma)) \ell(\gamma) / \sqrt{N-1})}{s_{K}(\ell(\gamma) / \sqrt{N-1})}\right\}^{N-1} \mu(A) d \Pi(\gamma) \\
\leq \int_{\Gamma} \mu(A) d \Pi(\gamma)=\mu(A)<\infty
\end{gathered}
$$

holds if $K \leq 0$, and that

$$
\begin{aligned}
\int_{\Gamma} \tau & (\ell(\gamma))\left\{\frac{s_{N-1}(\tau(\ell(\gamma)) \ell(\gamma) / \sqrt{N-1})}{s_{N-1}(\ell(\gamma) / \sqrt{N-1})}\right\}^{N-1} \mu(A) d \Pi(\gamma) \\
& =\int_{A} \tau\left(|x-y|_{X}\right)\left\{\frac{\sin \left(\tau\left(|x-y|_{X}\right)|x-y|_{X}\right)}{\sin \left(|x-y|_{X}\right)}\right\}^{N-1} d \mu(y) \\
& \leq \mu(A \cap B(x, \pi / 2))+\int_{A \cap A(x ; \pi / 2, \pi)}\left\{\frac{\sqrt{2} \sin \left(|x-y|_{X} / 2\right)}{\sin \left(|x-y|_{X}\right)}\right\}^{N-1} d \mu(y) \\
& \leq \mu(B(x, \pi / 2))+2^{(N+1) / 2} \int_{A(x ; \pi / 2, \pi)} \frac{1}{2}\left\{\frac{\sin \left(|x-y|_{X} / 2\right)}{\sin \left(|x-y|_{X}\right)}\right\}^{N-1} d \mu(y) \\
& \leq \mu(B(x, \pi / 2))+2^{(N+1) / 2} \mu(A(x ; \pi / 4, \pi / 2)) \\
& <\infty
\end{aligned}
$$

holds if $K=N-1>0$. We used the $(N-1, N)$-MCP in the fourth implication. This completes the proof.

Next we prove a key lemma in this section.

Lemma 4.2. Let $(X, \mu)$ satisfy the $(N-1, N)$-MCP. Then, for any $x \in X$ and $s, t \in[0, \pi / 2]$ with $s<t$, we have

$$
\mu(A(x ; s, t)) \geq \mu(A(x ; \pi-t, \pi-s)) .
$$

Proof. Take a large $M \in \mathbb{N}$ and set $\delta=(t-s) / M$ and $t_{m}:=s+m \delta$ for $0 \leq m \leq M$. For $1 \leq m \leq M$, we define a function $\tau_{m}:\left(\pi-t_{m}, \pi-t_{m-1}\right) \longrightarrow(0,1]$ by

$$
\tau_{m}(l):=\frac{l-\pi+t_{m-1}+t_{m}}{l} .
$$

Note that

$$
\begin{gathered}
\tau_{m}\left(\pi-t_{m}\right) \cdot\left(\pi-t_{m}\right)=t_{m-1}, \quad \tau_{m}\left(\pi-t_{m-1}\right) \cdot\left(\pi-t_{m-1}\right)=t_{m}, \\
\frac{d}{d l}\left[\tau_{m}(l) l\right]=1 .
\end{gathered}
$$


Applying Lemma 4.1 to each $A_{m}:=\left(x ; \pi-t_{m}, \pi-t_{m-1}\right)$ and $\tau_{m}$, we have

$$
\begin{aligned}
\mu(A(x ; s, t)) & =\sum_{m=1}^{M} \mu\left(A\left(x ; t_{m-1}, t_{m}\right)\right) \\
& \geq \sum_{m=1}^{M} \int_{\Gamma}\left\{\frac{s_{N-1}\left(\tau_{m}(\ell(\gamma)) \ell(\gamma) / \sqrt{N-1}\right)}{\boldsymbol{s}_{N-1}(\ell(\gamma) / \sqrt{N-1})}\right\}^{N-1} \mu\left(A_{m}\right) d \Pi_{x, A_{m}}(\gamma) \\
& =\sum_{m=1}^{M} \int_{A_{m}}\left\{\frac{\sin \left(\tau_{m}\left(|x-y|_{X}\right)|x-y|_{X}\right)}{\sin \left(|x-y|_{X}\right)}\right\}^{N-1} d \mu(y) \\
& =\sum_{m=1}^{M} \int_{A_{m}}\left\{\frac{\sin \left(|x-y|_{X}-\pi+t_{m-1}+t_{m}\right)}{\sin \left(|x-y|_{X}\right)}\right\}^{N-1} d \mu(y) \\
& =\sum_{m=1}^{M} \int_{A_{m}}\left[\left\{\frac{\sin \left(\pi-|x-y|_{X}\right)}{\sin \left(|x-y|_{X}\right)}\right\}^{N-1}+\theta(\delta)\right] d \mu(y) \\
& =\sum_{m=1}^{M} \mu\left(A_{m}\right)+\theta(\delta) \\
& \rightarrow \mu(A(x ; \pi-t, \pi-s))
\end{aligned}
$$

as $M$ diverges to the infinity.

Theorem 4.3. (Bonnet-Myers theorem) If a metric measure space $(X, \mu)$ satisfies the $(K, N)$-MCP for some $K>0$ and $N>1$, then we have diam $X \leq \pi \sqrt{(N-1) / K}$.

Proof. It suffices to consider the case of $K=N-1$. Suppose that there exist two points $x, y \in X$ with $|x-y|_{X}=\pi+\varepsilon$ for some $\varepsilon>0$. Since $X$ is a length space, for any small $\delta \in(0, \varepsilon)$, we can take a unit speed curve $\gamma:\left[0, \pi+\varepsilon+\delta^{\prime}\right] \longrightarrow X$ such that $\gamma(0)=x, \gamma\left(\pi+\varepsilon+\delta^{\prime}\right)=y$, and that $\delta^{\prime} \in[0, \delta]$. If we put $z \delta:=\gamma\left(\varepsilon+2 \delta+\delta^{\prime}\right)$, then we find

$$
\varepsilon+2 \delta \leq\left|x-z_{\delta}\right|_{X} \leq \varepsilon+2 \delta+\delta^{\prime}, \quad \pi-2 \delta-\delta^{\prime} \leq\left|z_{\delta}-y\right|_{X} \leq \pi-2 \delta .
$$

Put

$$
t:=\frac{\pi-\varepsilon-2 \delta-\delta^{\prime}}{\pi-\delta}, \quad A:=e_{t}\left(\operatorname{supp} \Pi_{z_{\delta}, B(y, \delta)}\right) .
$$

Then it follows from the $(N-1, N)$-MCP that

$$
\begin{aligned}
\mu(A) & \geq t\left\{\frac{\sin \left(t\left(\left|y-z_{\delta}\right|_{X}+\delta\right)\right)}{\sin \left(\left|y-z_{\delta}\right|_{X}-\delta\right)}\right\}^{N-1} \mu(B(y, \delta))\left(\left(e_{t}\right)_{*} \Pi_{z \delta, B(y, \delta)}\right)(A) \\
& \geq\left(1-\frac{\varepsilon+\delta+\delta^{\prime}}{\pi-\delta}\right)\left\{\frac{\sin \left(\pi-\varepsilon-2 \delta-\delta^{\prime}\right)}{\sin \left(\pi-3 \delta-\delta^{\prime}\right)}\right\}^{N-1} \mu(B(y, \delta))
\end{aligned}
$$




$$
\begin{aligned}
& \geq\left(1-\frac{\varepsilon+2 \delta}{\pi-\delta}\right)\left\{\frac{\sin (\pi-\varepsilon-3 \delta)}{\sin (\pi-4 \delta)}\right\}^{N-1} \mu(B(y, \delta)) \\
& =\left(1-\frac{\varepsilon+2 \delta}{\pi-\delta}\right)\left\{\frac{\sin (\varepsilon+3 \delta)}{\sin (4 \delta)}\right\}^{N-1} \mu(B(y, \delta)) .
\end{aligned}
$$

On one hand, we observe

$$
\begin{aligned}
A & \subset B\left(z_{\delta}, t\left(\left|z_{\delta}-y\right|_{X}+\delta\right)\right) \subset B\left(x, \varepsilon+2 \delta+\delta^{\prime}+t(\pi-\delta)\right) \\
& =B(x, \pi) .
\end{aligned}
$$

On the other hand, for any $\xi \in \operatorname{supp} \Pi_{z \delta}, B(y, \delta)$, we see

$$
\begin{aligned}
|x-\xi(t)|_{X} & \geq|x-\xi(1)|_{X}-(1-t) \ell(\xi) \\
& >(\pi+\varepsilon)-\delta-(1-t)\left(\left|z_{\delta}-y\right|_{X}+\delta\right) \\
& \geq \pi+\varepsilon-\delta-(1-t)(\pi-\delta) \\
& =\pi+\varepsilon-\delta-\left(\varepsilon+\delta+\delta^{\prime}\right) \\
& =\pi-2 \delta-\delta^{\prime} \\
& \geq \pi-3 \delta
\end{aligned}
$$

and hence $A \subset B(x, \pi) \backslash \bar{B}(x, \pi-3 \delta)$. Thus we have, by Lemma 4.2 and the doubling condition (Lemma 2.5 with $K=0$ ),

$$
\mu(A) \leq \mu(A(x ; \pi-3 \delta, \pi)) \leq \mu(B(x, 3 \delta)) \leq 3^{N} \mu(B(x, \delta)) .
$$

Therefore we obtain, since $N>1$,

$$
\frac{\mu(B(x, \delta))}{\mu(B(y, \delta))} \geq 3^{-N}\left(1-\frac{\varepsilon+2 \delta}{\pi-\delta}\right)\left\{\frac{\sin (\varepsilon+3 \delta)}{\sin 4 \delta}\right\}^{N-1} \rightarrow \infty
$$

as $\delta$ tends to zero. However, this is a contradiction because we can exchange the roles of $x$ and $y$.

Recall that we set $S(x, r)=\left\{y \in X|| x-\left.y\right|_{X}=r\right\}$ for $x \in X$ and $r>0$.

Lemma 4.4. Let $(X, \mu)$ satisfy the $(N-1, N)$-MCP for some $N>1$.

(i) For every $x \in X$, the set $S(x, \pi)$ has a null measure.

(ii) If $x, y \in X$ satisfies $|x-y|_{X}=\pi$, then we have, for any $\varepsilon \in(0, \pi / 2)$,

$$
\mu(B(x, \varepsilon))=\mu(B(y, \varepsilon)) .
$$


Proof. (i) We can suppose that $S(x, \pi) \neq \emptyset$, in particular, $X$ contains more than two points. Fix an arbitrary $\varepsilon>0$ and let $\left\{x_{i}\right\}_{i=1}^{M}$ be a maximal $2 \varepsilon$-discrete set in $S(x, 3 \varepsilon)$, i.e., $\left\{x_{i}\right\}_{i=1}^{M} \subset S(x, 3 \varepsilon),\left|x_{i}-x_{j}\right|_{X} \geq 2 \varepsilon$ holds if $i \neq j$, and $\left\{B\left(x_{i}, 2 \varepsilon\right)\right\}_{i=1}^{M}$ covers $S(x, 3 \varepsilon)$. Note that $B\left(x_{i}, \varepsilon\right)$ 's are mutually disjoint. For any $y \in S(x, \pi)$, there exists a point $z \in S(x, 3 \varepsilon)$ such that $|y-z|_{X}<\pi-2 \varepsilon$, and $\left|z-x_{i}\right|_{X}<2 \varepsilon$ holds for some $i$. For such $i$, we observe

$$
\begin{aligned}
& \left|y-x_{i}\right|_{X} \leq|y-z|_{X}+\left|z-x_{i}\right|_{X}<\pi, \\
& \left|y-x_{i}\right|_{X} \geq|y-x|_{X}-\left|x-x_{i}\right|_{X}=\pi-3 \varepsilon .
\end{aligned}
$$

Namely, we see $y \in A\left(x_{i} ; \pi-3 \varepsilon, \pi\right)$. Combining this with Lemma 4.2, we obtain

$$
\begin{aligned}
\mu(S(x, \pi)) & \leq \mu\left(\bigcup_{i=1}^{M} A\left(x_{i} ; \pi-3 \varepsilon, \pi\right)\right) \\
& \leq \sum_{i=1}^{M} \mu\left(A\left(x_{i} ; \pi-3 \varepsilon, \pi\right)\right) \leq \sum_{i=1}^{M} \mu\left(B\left(x_{i}, 3 \varepsilon\right)\right) \\
& \leq 3^{N} \sum_{i=1}^{M} \mu\left(B\left(x_{i}, \varepsilon\right)\right)=3^{N} \mu\left(\bigcup_{i=1}^{M} B\left(x_{i}, \varepsilon\right)\right) \\
& \leq 3^{N} \mu(B(x, 4 \varepsilon)) \rightarrow 0
\end{aligned}
$$

as $\varepsilon$ tends to zero by Corollary 2.6. This completes the proof.

(ii) It is a straightforward corollary to Lemma 4.2 through Theorem 4.3 and (i) of this lemma. Indeed, we have

$$
\mu(B(x, \varepsilon)) \geq \mu(A(x ; \pi-\varepsilon, \pi))=\mu(X \backslash B(x, \pi-\varepsilon)) \geq \mu(B(y, \varepsilon)) .
$$

The converse inequality is obtained similarly.

We remark that Lemma 4.4(i) is not covered by Lemma 2.5. Now we obtain a result concerning the maximal diameter situation.

Theorem 4.5. If a metric measure space $(X, \mu)$ satisfies the $(K, N)$-MCP for some $K>0$ and $N>1$, then, for any $x \in X$, the set $S(x, \pi \sqrt{(N-1) / K})$ consists of at most one point.

Proof. Suppose that $K=N-1$ and that there exist two points $y, z \in S(x, \pi)$ satisfying $\varepsilon:=|y-z|_{X} / 2>0$. Then, by Lemma 4.4, Theorem 4.3, and by Lemma 4.2, we obtain

$$
\begin{aligned}
2 \mu(B(x, \varepsilon)) & =\mu(B(y, \varepsilon))+\mu(B(z, \varepsilon))=\mu(B(y, \varepsilon) \cup B(z, \varepsilon)) \\
& \leq \mu(A(x ; \pi-\varepsilon, \pi)) \leq \mu(B(x, \varepsilon)) .
\end{aligned}
$$

This contradicts to $\mu(B(x, \varepsilon))>0$, and hence we complete the proof. 


\section{Bishop-Gromov volume comparison theorem}

This section is devoted to proving an analogue of the Bishop-Gromov volume comparison theorem. See [Cl, Theorem 3.10] for the Riemannian case.

Theorem 5.1. (Bishop-Gromov volume comparison theorem) Let $(X, \mu)$ be a metric space satisfying the $(K, N)$-MCP. Then, for any $x \in X$, the function

$$
\mu(B(x, r)) /\left\{\int_{0}^{r} s_{K}\left(\frac{s}{\sqrt{N-1}}\right)^{N-1} d s\right\}
$$

is monotone non-increasing in $r \in(0, \infty)(r \in(0, \pi \sqrt{(N-1) / K})$ if $K>0)$.

Proof. The proof is based on the discretization of that in the Riemannian case (roughly speaking, the integration of the Bishop inequality). Take $r>0$. By Theorems 4.3 and 4.5, we can suppose $r \leq \pi \sqrt{(N-1) / K}$ if $K>0$. For a small $t \in(0,1)$ and any $l, m \in \mathbb{N}$ with $l<m$, it follows from the $(K, N)$-MCP with $x=x$, $A=A\left(x ; t^{l} r, t^{l-1} r\right)$, and $t=t^{m-l}$ that

$$
\begin{aligned}
& \mu\left(A\left(x ; t^{m} r, t^{m-1} r\right)\right) \\
& \geq t^{m-l} \inf _{t \leq s \leq 1}\left\{\frac{s_{K}\left(s t^{m-1} r / \sqrt{N-1}\right)}{s_{K}\left(s t^{l-1} r / \sqrt{N-1}\right)}\right\}^{N-1} \mu\left(A\left(x ; t^{l} r, t^{l-1} r\right)\right) \\
& \geq t^{m-l}\left[\left\{\inf _{t \leq s \leq 1} s_{K}\left(\frac{s t^{m-1} r}{\sqrt{N-1}}\right)\right\} /\left\{\sup _{t \leq s \leq 1} s_{K}\left(\frac{s t^{l-1} r}{\sqrt{N-1}}\right)\right\}\right]^{N-1} \\
& \times \mu\left(A\left(x ; t^{l} r, t^{l-1} r\right)\right) .
\end{aligned}
$$

Thus we have, for all $l \leq j \leq m-1$,

$$
\begin{aligned}
& \mu\left(A\left(x ; t^{j} r, t^{j-1} r\right)\right) \sum_{i=m}^{\infty} t^{i} \inf _{t \leq s \leq 1} s_{K}\left(s t^{i-1} r / \sqrt{N-1}\right)^{N-1} \\
& \leq\left\{\sum_{i=m}^{\infty} \mu\left(A\left(x ; t^{i} r, t^{i-1} r\right)\right)\right\} t^{j} \sup _{t \leq s \leq 1} s_{K}\left(s t^{j-1} r / \sqrt{N-1}\right)^{N-1} \\
& =\mu\left(B\left(x, t^{m-1} r\right)\right) t^{j} \sup _{t \leq s \leq 1} s_{K}\left(s t^{j-1} r / \sqrt{N-1}\right)^{N-1} .
\end{aligned}
$$


Therefore we obtain

$$
\begin{aligned}
\mu & \left(B\left(x, t^{l-1} r\right)\right) \\
= & \mu\left(B\left(x, t^{m-1} r\right)\right)+\sum_{j=l}^{m-1} \mu\left(A\left(x ; t^{j} r, t^{j-1} r\right)\right) \\
\leq & {\left[1+\left\{\sum_{j=l}^{m-1} t^{j} \sup _{t \leq s \leq 1} s_{K}\left(\frac{s t^{j-1} r}{\sqrt{N-1}}\right)^{N-1}\right\} /\left\{\sum_{i=m}^{\infty} t^{i} \inf _{t \leq s \leq 1} s_{K}\left(\frac{s t^{i-1} r}{\sqrt{N-1}}\right)^{N-1}\right\}\right] } \\
& \times \mu\left(B\left(x, t^{m-1} r\right)\right) \\
\leq & {\left[\left\{\sum_{j=l}^{\infty} t^{j} \sup _{t \leq s \leq 1} s_{K}\left(\frac{s t^{j-1} r}{\sqrt{N-1}}\right)^{N-1}\right\} /\left\{\sum_{i=m}^{\infty} t^{i} \inf _{t \leq s \leq 1} s_{K}\left(\frac{s t^{i-1} r}{\sqrt{N-1}}\right)^{N-1}\right\}\right] } \\
& \times \mu\left(B\left(x, t^{m-1} r\right)\right),
\end{aligned}
$$

and hence

$$
\begin{aligned}
& \mu\left(B\left(x, t^{l-1} r\right)\right) /\left\{\sum_{j=l}^{\infty}\left(t^{j-1} r-t^{j} r\right) \sup _{t \leq s \leq 1} s_{K}\left(\frac{s t^{j-1} r}{\sqrt{N-1}}\right)^{N-1}\right\} \\
& \quad \leq \mu\left(B\left(x, t^{m-1} r\right)\right) /\left\{\sum_{i=m}^{\infty}\left(t^{i-1} r-t^{i} r\right) \inf _{t \leq s \leq 1} s_{K}\left(\frac{s t^{i-1} r}{\sqrt{N-1}}\right)^{N-1}\right\} .
\end{aligned}
$$

This completes the proof by letting $t$ tend to 1 as well as $l$ and $m$ go to the infinity.

\section{Stability and compactness}

In this section, we consider the behavior of the $(K, N)$-MCP under the measured Gromov-Hausdorff convergence. The Wasserstein space will play a crucial role. See [F] and [KS2] for the measured Gromov-Hausdorff convergence, and see [LV], [S2], and $[\mathrm{V}]$ for the Wasserstein space.

6.1. Measured Gromov-Hausdorff topology. We first recall the Gromov-Hausdorff distance between compact metric spaces. See [G] for more details. For two closed subsets $A$ and $A^{\prime}$ in a metric space $Z$, the Hausdorff distance $d_{H}^{Z}$ between them is defined by

$$
d_{H}^{Z}\left(A, A^{\prime}\right):=\inf \left\{\varepsilon>0 \mid A \subset B\left(A^{\prime}, \varepsilon\right), A^{\prime} \subset B(A, \varepsilon)\right\} .
$$

More generally, for two compact metric spaces $X$ and $Y$, we define the GromovHausdorff distance $d_{G H}$ between them by

$$
d_{G H}(X, Y):=\inf _{Z, \varphi, \psi} d_{H}^{Z}(\varphi(X), \psi(Y)),
$$


where the infimum is taken over all metric spaces $Z$ and isometric embeddings $\varphi: X \longrightarrow Z$ and $\psi: Y \longrightarrow Z$. If we denote by $\mathcal{C}$ the isometric classes of compact metric spaces, then $\left(\mathcal{C}, d_{G H}\right)$ is a complete metric space. The topology of $\mathcal{C}$ induced from $d_{G H}$ is called the Gromov-Hausdorff topology. It is convenient to estimate the Gromov-Hausdorff distance in terms of the $\varepsilon$-approximating map. For metric spaces $X$ and $X^{\prime}$, a (not necessarily continuous) map $\varphi: X \longrightarrow X^{\prime}$ is called an $\varepsilon$-approximating map for $\varepsilon \geq 0$ if it satisfies $\bar{B}^{X^{\prime}}(\varphi(X), \varepsilon) \supset X^{\prime}$ and if

$$
|| \varphi(x)-\left.\varphi(y)\right|_{X^{\prime}}-|x-y|_{X} \mid \leq \varepsilon
$$

holds for all $x, y \in X$. Note that a 0 -approximating map is nothing but an isometry.

Lemma 6.1. Let $X, Y \in \mathcal{C}$ and $\varepsilon>0$.

(i) If $d_{G H}(X, Y)<\varepsilon$, then there exists a $2 \varepsilon$-approximating map from $X$ to $Y$.

(ii) If there exists an $\varepsilon$-approximating map from $X$ to $Y$, then $d_{G H}(X, Y) \leq 2 \varepsilon$.

In particular, a sequence $\left\{X_{i}\right\}_{i=1}^{\infty} \subset \mathcal{C}$ converges to $X \in \mathcal{C}$ if and only if there exists a sequence of $\varepsilon_{i}$-approximating maps $\varphi_{i}: X_{i} \longrightarrow X$ with $\lim _{i \rightarrow \infty} \varepsilon_{i}=0$. For the later use, we recall an easily proved lemma.

Lemma 6.2. Let $\left\{X_{i}\right\}_{i=1}^{\infty} \subset \mathcal{C}$ be a sequence of compact, geodesic metric spaces converging to a compact metric space $X \in \mathcal{C}$ in the Gromov-Hausdorff topology with a sequence $\left\{\varepsilon_{i}\right\}_{i=1}^{\infty}$ tending to zero and $\varepsilon_{i}$-approximating maps $\left\{\varphi_{i}\right\}_{i=1}^{\infty}$. For a sequence of minimal geodesics $\gamma_{i}:[0,1] \longrightarrow X_{i}, i \in \mathbb{N}$, if the sequences of end points $\left\{\varphi_{i}\left(\gamma_{i}(0)\right)\right\}_{i=1}^{\infty}$ and $\left\{\varphi_{i}\left(\gamma_{i}(1)\right)\right\}_{i=1}^{\infty}$ converge to some points $x, y \in X$, respectively, then a subsequence of $\left\{\varphi_{i} \circ \gamma_{i}\right\}_{i=1}^{\infty}$ converges to a minimal geodesic from $x$ to y uniformly.

We next recall the measured Gromov-Hausdorff convergence introduced in $[\mathrm{F}]$.

Definition 6.3. (Measured Gromov-Hausdorff convergence, $[\mathrm{F}]$ ) A directed system of metric measure spaces $\left\{\left(X_{\alpha}, \mu_{\alpha}\right)\right\}_{\alpha \in \mathcal{A}}$ is said to converge to a metric measure space $(X, \mu)$ in the sense of the measured Gromov-Hausdorff convergence if there exists a directed system of positive numbers $\left\{\varepsilon_{\alpha}\right\}_{\alpha \in \mathcal{A}}$ satisfying the following conditions:

(1) $\left\{\varepsilon_{\alpha}\right\}_{\alpha \in \mathcal{A}}$ converges to zero;

(2) For each $\alpha \in \mathcal{A}$, we have a Borel, measurable, and $\varepsilon_{\alpha}$-approximating map $\varphi_{\alpha}: X_{\alpha} \longrightarrow X$

(3) A directed system of push-forward measures $\left\{\left(\varphi_{\alpha}\right)_{*}\left(\mu_{\alpha}\right)\right\}_{\alpha}$ converges to $\mu$ weakly, i.e., for any $f \in C(X)$, we have

$$
\lim _{\alpha \in \mathcal{A}} \int_{X} f d\left(\left(\varphi_{\alpha}\right)_{*}\left(\mu_{\alpha}\right)\right)=\int_{X} f d \mu .
$$

Here $C(X)$ denotes the set of all continuous functions on $X$. 
If we define $\mathcal{C} \mathcal{M}$ as the isomorphic classes of all compact metric spaces equipped with Radon measures, then the measured Gromov-Hausdorff convergence gives a topology on $\mathcal{C} \mathcal{M}$, and we call it the measured Gromov-Hausdorff topology. We know that this topology is Hausdorff ([F, Proposition 2.7]) and that the projection $\mathcal{C} \mathcal{M}(V) \longrightarrow \mathcal{C}$ is proper, where we set

$$
\mathcal{C} \mathcal{M}(V):=\{(X, \mu) \in \mathcal{C} \mathcal{M} \mid \mu(X) \leq V\}
$$

for $V>0$ ([F, Proposition 2.10]). For $K \in \mathbb{R}, N \geq 1, V>0$, and $D>0$, we define $\mathcal{C} \mathcal{M}(K, N, V, D) \subset \mathcal{C} \mathcal{M}(V)$ as the isomorphic classes of compact metric measure spaces $(X, \mu)$ in $\mathcal{C} \mathcal{M}(V)$ satisfying the $(K, N)$-MCP and diam $X \leq D$. The following is an easy corollary of Gromov's precompactness theorem ([G, Proposition 5.2]) by virtue of Theorem 5.1.

Theorem 6.4. Let $\left\{\left(X_{i}, \mu_{i}\right)\right\}_{i=1}^{\infty} \subset \mathcal{C} \mathcal{M}(K, N, V, D)$. Then it has a subsequence which is convergent in the measured Gromov-Hausdorff topology.

If we denote by $(X, \mu) \in \mathcal{C} \mathcal{M}$ that limit space, then we immediately observe $\mu(X) \leq V$ and diam $X \leq D$. To show that $(X, \mu)$ also satisfies the $(K, N)$-MCP, we need to recall the Wasserstein space and some results in [LV].

6.2. Wasserstein spaces. Let $X$ be a complete, separable metric measure space, and recall that $\mathcal{P}^{2}(X)$ denotes the set of all Borel probability measures, say $\mu$, satisfying $\int_{X}|x-y|_{X}^{2} d \mu(y)<\infty$ for some (and hence all) $x \in X$. Given two probability measures $\mu, v \in \mathcal{P}^{2}(X)$, a Borel measure $q$ on $X \times X$ is called a coupling of $\mu$ and $v$ if, for any measurable set $A \subset X$, we have $q(A \times X)=\mu(A)$ and $q(X \times A)=v(A)$. For example, the product measure $\mu \times v$ is a coupling of $\mu$ and $\nu$. We define the $L^{2}$-Wasserstein distance $d_{W}$ on $\mathcal{P}^{2}(X)$ by

$$
d_{W}(\mu, v):=\inf \left\{\left(\int_{X \times X}|x-y|_{X}^{2} d q(x, y)\right)^{1 / 2} \mid q: \text { coupling of } \mu \text { and } v\right\}
$$

for $\mu, v \in \mathscr{P}^{2}(X)$, and we call $\left(\mathscr{P}^{2}(X), d_{W}\right)$ the $L^{2}$-Wasserstein space over $X$. Then $\left(\mathscr{P}^{2}(X), d_{W}\right)$ is a complete and separable metric space (see [S2, Proposition 2.10]). Furthermore, $\left(\mathcal{P}^{2}(X), d_{W}\right)$ is compact or a length space if and only if so is $X$, respectively. In particular, if $X$ is compact and geodesic, then so is $\left(\mathcal{P}^{2}(X), d_{W}\right)$.

Proposition 6.5 (cf. [V, Theorem 7.12]). A sequence $\left\{\mu_{i}\right\}_{i=1}^{\infty} \subset \mathcal{P}^{2}(X)$ converges to $\mu \in \mathcal{P}^{2}(X)$ with respect to $d_{W}$ if and only if $\mu_{i}$ converges to $\mu$ weakly and

$$
\lim _{R \rightarrow \infty} \sup _{i \in \mathbb{N}} \int_{X \backslash B(x, R)}|x-y|_{X}^{2} d \mu_{i}(y)=0
$$

holds for some (and hence every) point $x \in X$. 
We observe that (6.1) automatically holds true if $X$ is bounded. The following two results obtained in [LV] will play key roles in our discussions.

Proposition 6.6 ([LV, Proposition 4.1, Corollary 4.3]). If $\varphi: X \longrightarrow X^{\prime}$ is a Borel, $\varepsilon$ approximating map, then $\varphi_{*}:\left(\mathscr{P}^{2}(X), d_{W}\right) \longrightarrow\left(\mathscr{P}^{2}\left(X^{\prime}\right), d_{W}\right)$ is $\tilde{\varepsilon}$-approximating with

$$
\tilde{\varepsilon}=4 \varepsilon+\left\{\varepsilon\left(2 \operatorname{diam} X^{\prime}+\varepsilon\right)\right\}^{1 / 2} .
$$

In particular, if a sequence of compact metric spaces $\left\{X_{i}\right\}_{i=1}^{\infty}$ converges to a compact metric space $X$ in the Gromov-Hausdorff topology equipped with Borel, $\varepsilon_{i}$ approximating maps $\varphi_{i}, i \in \mathbb{N}$, then the sequence $\left\{\left(\mathcal{P}^{2}\left(X_{i}\right), d_{W}\right)\right\}_{i=1}^{\infty}$ converges to $\left(\mathscr{P}^{2}(X), d_{W}\right)$ in the Gromov-Hausdorff topology with $\tilde{\varepsilon}_{i}$-approximating maps $\left(\varphi_{i}\right)_{*}$.

Proposition 6.7 ([LV, Proposition 2.10]). Let X be a compact geodesic metric space. Then any minimal geodesic in $\left(\mathcal{P}^{2}(X), d_{W}\right)$ is given by the displacement interpolation associated to some dynamical transference plan.

6.3. Stability and compactness. Now we prove the stability of the $(K, N)$-MCP under the measured Gromov-Hausdorff convergence. The idea of the proof is as follows. If we consider the dynamical transference plan $\Pi=\Pi_{x, A}$ as a family of geodesics in $X$, then, as it contains uncountably many geodesics, it is impossible to control the behaviors of all of them simultaneously. However, we can regard it as one geodesic from $\delta_{x}$ to $\left(\left.\mu\right|_{A}\right)^{-}$in the Wassestein space $\left(\mathcal{P}^{2}(X), d_{W}\right)$, and then usual techniques are applicable. All spaces in this subsection are assumed to be compact.

Theorem 6.8 (Stability). A measured Gromov-Hausdorff limit, with a positive total mass, of a sequence of metric measure spaces satisfying the $(K, N)$-MCP also satisfies the $(K, N)-M C P$.

Proof. We first assume $K \leq 0$. Let $\left\{\left(X_{i}, \mu_{i}\right)\right\}_{i=1}^{\infty} \subset \mathcal{C} \mathcal{M}$ be a sequence of metric measure spaces satisfying the $(K, N)$-MCP. We suppose that it converges to some metric measure space $(X, \mu)$ with $\mu(X)>0$ in the measured Gromov-Hausdorff topology, so that we have a sequence $\left\{\varepsilon_{i}\right\}_{i=1}^{\infty}$ tending to zero and a Borel, measurable, and $\varepsilon_{i}$-approximating map $\varphi_{i}: X_{i} \longrightarrow X, i \in \mathbb{N}$, as in Definition 6.3.

Fix a point $x \in X$ and a measurable set $A \subset X$ with $\mu(A)>0$. For each (large) $i \in \mathbb{N}$, we choose a point $x_{i} \in \varphi_{i}^{-1}\left(\bar{B}^{X}\left(x, \varepsilon_{i}\right)\right)$ and put $A_{i}:=\varphi_{i}^{-1}(A)$. We remark that, by the definition of the $\varepsilon_{i}$-approximating map, $\varphi_{i}^{-1}\left(\bar{B}^{X}\left(x, \varepsilon_{i}\right)\right)$ is not an empty set. Moreover, as $\mu(A)>0$, we know $\mu_{i}\left(A_{i}\right)=\left(\left(\varphi_{i}\right)_{*} \mu_{i}\right)(A)>0$ and hence $A_{i}$ in not empty for large $i$. By the $(K, N)$-MCP, for each $i \in \mathbb{N}$, we have a dynamical transference plan $\Pi_{i}=\Pi_{x_{i}, A_{i}}$ such that the displacement interpolation associated to it satisfies the conditions (1) and (2) in Definition 2.1. Note that

$$
\left(\varphi_{i}\right)_{*}\left(\left(e_{0}\right)_{*} \Pi_{i}\right)=\left(\varphi_{i}\right)_{*} \delta_{x_{i}}=\delta_{\varphi_{i}\left(x_{i}\right)} \rightarrow \delta_{x}
$$


and, by Proposition 6.5,

$$
\left(\varphi_{i}\right)_{*}\left(\left(e_{1}\right)_{*} \Pi_{i}\right)=\left(\varphi_{i}\right)_{*}\left(\left.\mu_{i}\right|_{A_{i}}\right)^{-}=\left(\left.\left[\left(\varphi_{i}\right)_{*}\left(\mu_{i}\right)\right]\right|_{A}\right)^{-} \rightarrow\left(\left.\mu\right|_{A}\right)^{-}
$$

in $\left(\mathcal{P}^{2}(X), d_{W}\right)$ as $i$ diverges to the infinity, respectively. Thus it follows from Lemma 6.2 and Proposition 6.6 that a subsequence of $\left\{\left(\varphi_{i}\right)_{*}\left[\left(e_{t}\right)_{*} \Pi_{i}\right]\right\}_{t \in[0,1]}, i \in$ $\mathbb{N}$, converges to a minimal geodesic $\left\{v_{t}\right\}_{t \in[0,1]}$ between $\delta_{x}$ and $\left(\left.\mu\right|_{A}\right)^{-}$. Again we denote this convergent subsequence by $\left\{\left(\varphi_{i}\right)_{*}\left[\left(e_{t}\right)_{*} \Pi_{i}\right]\right\}_{t \in[0,1]}, i \in \mathbb{N}$. Moreover, Proposition 6.7 implies that $\left\{v_{t}\right\}_{t \in[0,1]}$ is the displacement interpolation associated to some dynamical transference plan $\Pi=\Pi_{x, A}$ which clearly satisfies $\left(e_{0}\right)_{*} \Pi=\delta_{x}$ and $\left(e_{1}\right)_{*} \Pi=\left(\left.\mu\right|_{A}\right)^{-}$.

Now we consider the condition (2) in Definition 2.1. We fix $t \in(0,1)$ and put

$$
\begin{aligned}
d \nu_{i} & :=\left(e_{t}\right)_{*}\left(t\left\{\frac{s_{K}(t \ell(\gamma) / \sqrt{N-1})}{s_{K}(\ell(\gamma) / \sqrt{N-1})}\right\}^{N-1} \mu_{i}\left(A_{i}\right) d \Pi_{i}(\gamma)\right), \\
d \nu & :=\left(e_{t}\right)_{*}\left(t\left\{\frac{s_{K}(t \ell(\gamma) / \sqrt{N-1})}{s_{K}(\ell(\gamma) / \sqrt{N-1})}\right\}^{N-1} \mu(A) d \Pi(\gamma)\right)
\end{aligned}
$$

on $X_{i}$ and $X$, respectively. Since $\left(\varphi_{i}\right)_{*}\left[\left(e_{t}\right)_{*} \Pi_{i}\right]$ converges to $\left(e_{t}\right)_{*} \Pi$ weakly and $X_{i}$ converges to $X$ in the Gromov-Hausdorff topology, we find that $\left(\varphi_{i}\right)_{*}\left(v_{i}\right)$ converges to $v$ weakly as $i$ diverges to the infinity. The $(K, N)$-MCP of $\left(X_{i}, \mu_{i}\right)$ yields that $\mu_{i} \geq v_{i}$ holds as measures for every $i$. Therefore we have $\mu \geq v$ and hence $(X, \mu)$ satisfies the $(K, N)$-MCP. This completes the proof in the case of $K \leq 0$.

If $K>0$, then we take $A \subset B^{X}(x, \pi \sqrt{(N-1) / K})$ and set, for each $i \in \mathbb{N}$,

$$
A_{i}:=\varphi_{i}^{-1}(A) \cap B^{X_{i}}\left(x_{i}, \pi \sqrt{(N-1) / K}\right) .
$$

Then a completely similar discussion proves the theorem.

Combining this stability with Theorem 6.4, we obtain the compactness of the family

$$
\mathcal{C} \mathcal{M}\left(K, N, V^{\prime}, V, D\right):=\left\{X \in \mathcal{C} \mathcal{M}(K, N, V, D) \mid \mu(X) \geq V^{\prime}\right\},
$$

where $0<V^{\prime} \leq V$.

Theorem 6.9 (Compactness). For any $K \in \mathbb{R}, N \geq 1, V \geq V^{\prime}>0$, and any $D>0$, the set $\mathcal{C} \mathcal{M}\left(K, N, V^{\prime}, V, D\right)$ is compact in the measured Gromov-Hausdorff topology.

In particular, the family $\mathcal{C} \mathcal{M}(K, N, 1,1, D)$ (i.e., spaces with probability measures) is compact. 
6.4. Non-compact case. The discussion in the previous subsection is also applicable to the non-compact case by weakening the measured Gromov-Hausdorff convergence to the pointed one. We suppose that all metric spaces appearing in this subsection are complete.

Definition 6.10 (Pointed measured Gromov-Hausdorff convergence). A directed system of pointed metric measure spaces $\left\{\left(X_{\alpha}, \mu_{\alpha}, z_{\alpha}\right)\right\}_{\alpha \in \mathcal{A}}$ is said to converge to a pointed metric measure space $(X, \mu, z)$ in the sense of the pointed measured GromovHausdorff convergence if there exist two directed systems $\left\{\varepsilon_{\alpha}\right\}_{\alpha \in \mathcal{A}}$ and $\left\{r_{\alpha}\right\}_{\alpha \in \mathcal{A}}$ satisfying the following:

(1) $\left\{\varepsilon_{\alpha}\right\}_{\alpha \in \mathcal{A}}$ tends to zero and $\left\{r_{\alpha}\right\}_{\alpha \in \mathcal{A}}$ diverges to the infinity;

(2) For each $\alpha \in \mathcal{A}$, we have a Borel, measurable, and $\varepsilon_{\alpha}$-approximating map $\varphi_{\alpha}: B^{X_{\alpha}}\left(z_{\alpha}, r_{\alpha}\right) \longrightarrow B^{X}\left(z, r_{\alpha}\right)$

(3) A directed system of push-forward measures $\left\{\left(\varphi_{\alpha}\right)_{*}\left(\mu_{\alpha}\right)\right\}_{\alpha \in \mathcal{A}}$ converges to $\mu$ vaguely, i.e., for any $f \in C_{0}(X)$, we have

$$
\lim _{\alpha \in \mathcal{A}} \int_{X} f d\left(\left(\varphi_{\alpha}\right)_{*}\left(\mu_{\alpha}\right)\right)=\int_{X} f d \mu .
$$

Here $C_{0}(X)$ denotes the set of all continuous functions on $X$ whose supports are compact.

Theorem 6.11. A pointed measured Gromov-Hausdorff limit, with positive total mass, of a sequence of pointed metric measure spaces satisfying the $(K, N)-M C P$ also satisfies the $(K, N)-M C P$.

Proof. Take a point $x \in X$ and a measurable set $A \subset X$. As $X$ is proper, we can apply the discussion in the proof of Theorem 6.8 to each $A \cap B(x, m), m \in \mathbb{N}$. This completes the proof.

\section{References}

[ABN] A. D. Alexandrov, V. N. Berestovskii, and I. G. Nikolaev, Generalized Riemannian spaces. Russian Math. Surveys 41 (1986), 1-54. Zbl 0625.53059 MR 0854238

[AT] L. Ambrosio and P. Tilli, Topics on analysis in metric spaces. Oxford University Press, Oxford 2004. Zbl 1080.28001 MR 2039660

[BC] R. L. Bishop and R. J. Crittenden, Geometry of manifolds. Pure Appl. Math. 15, Academic Press, New York, London 1964. Zbl 0132.16003 MR 0169148

[BBI] D. Burago, Yu. Burago, and S. Ivanov, A course in metric geometry. Grad. Stud. Math. 33, Amer. Math. Soc., Providence, RI, 2001. Zbl 0981.51016 MR 1835418 
[BGP] Yu. Burago, M. Gromov, and G. Perel'man, A. D. Aleksandrov spaces with curvatures bounded below. Uspekhi Mat. Nauk 47 (1992), 3-52; English transl. Russian Math. Surveys 47 (1992), 1-58. Zbl 0802.53018 MR 1185284

[Cl] I. Chavel, Riemannian geometry-a modern introduction. Cambridge Tracts in Math. 108, Cambridge University Press, Cambridge 1993. Zbl 0810.53001 MR 1271141

[CC] J. Cheeger and T. H. Colding, On the structure of spaces with Ricci curvature bounded below. I; II; III. J. Differential Geom. 46 (1997), 406-480; ibid. 54 (2000), 13-35; ibid. 54 (2000), 37-74. Zbl 0902.53034 MR 1484888; Zbl 1027.53042 MR 1815410; Zbl 1027.53043 MR 1815411

[F] K. Fukaya, Collapsing of Riemannian manifolds and eigenvalues of Laplace operator. Invent. Math. 87 (1987), 517-547. Zbl 0589.58034 MR 0874035

[G] M. Gromov, Metric structures for Riemannian and non-Riemannian spaces. Progr. Math. 152, Birkhäuser, Boston, MA, 1999. Zbl 0953.53002 MR 1699320

[KS1] K. Kuwae and T. Shioya, On generalized measure contraction property and energy functionals over Lipschitz maps. Potential Anal. 15 (2001), 105-121. Zbl 0996.31006 MR 1838897

[KS2] K. Kuwae and T. Shioya, Convergence of spectral structures: a functional analytic theory and its applications to spectral geometry. Comm. Anal. Geom. 11 (2003), 599-673. Zbl 1092.53026 MR 2015170

[LV] J. Lott and C. Villani, Ricci curvature for metric-measure spaces via optimal transport. Ann. of Math., to appear.

[M] S. B. Myers, Riemannian manifolds with positive mean curvature. Duke Math. J. 8 (1941), 401-404. Zbl 0025.22704 MR 0004518

[O] S. Ohta, Reconstructions of distances by energy forms. Proc. Amer. Math. Soc. 134 (2006), 3405-3415. Zbl 1109.58013 MR 2231926

[R1] A. Ranjbar-Motlagh, A note on the Poincaré inequality. Studia Math. 154 (2003), 1-11. Zbl 1037.26012 MR 1949045

[R2] A. Ranjbar-Motlagh, Poincaré inequality for abstract spaces. Bull. Austral. Math. Soc. 71 (2005), 193-204. Zbl 02170493 MR 2133404

[RS] M.-K. von Renesse and K.-T. Sturm, Transport inequalities, gradient estimates, entropy and Ricci curvature. Comm. Pure Appl. Math. 58 (2005), 1-18. Zbl 1078.53028 MR 2142879

[S1] K.-T. Sturm, Diffusion processes and heat kernels on metric spaces. Ann. Probab. 26 (1998), 1-55. Zbl 0936.60074 MR 1617040

[S2] K.-T. Sturm, On the geometry of metric measure spaces. I. Acta Math. 196 (2006), 65-131. Zbl 1105.53035 MR 2237206

[S3] K.-T. Sturm, On the geometry of metric measure spaces. II. Acta Math. 196 (2006), 133-177. Zbl 1106.53032 MR 2237207

[V] C. Villani, Topics in optimal transportation. Grad. Stud. Math. 58, Amer. Math. Soc., Providence, RI, 2003. Zbl 1106.90001 MR 1964483

Received June 13, 2005

Shin-ichi Ohta, Department of Mathematics, Faculty of Science, Kyoto University, Kyoto 606-8502, Japan

E-mail: sohta@math.kyoto-u.ac.jp 